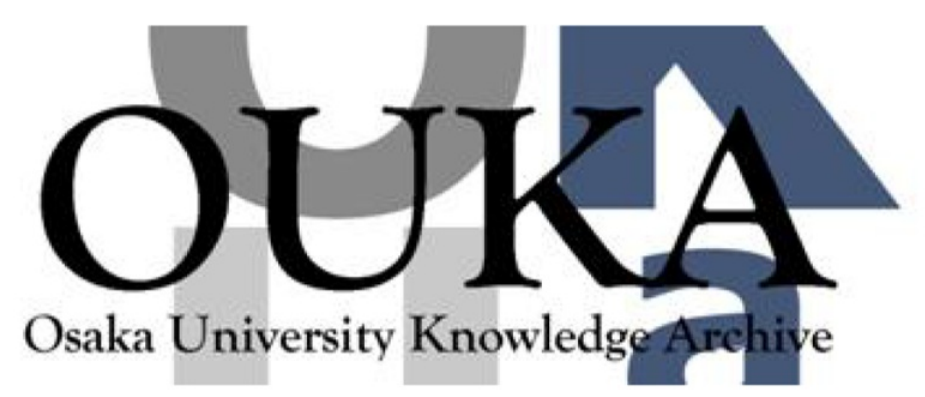

\begin{tabular}{|c|l|}
\hline Title & $\begin{array}{l}\text { Thermal energy of the crystalline one-component } \\
\text { plasma from dynamical simulations }\end{array}$ \\
\hline Author(s) & Farouki, R. T.; Hamaguchi, S. \\
\hline Citation & Physical Review E. 47(6) p. 4330-p. 4336 \\
\hline Issue Date & $1993-06$ \\
\hline oaire:version & VoR \\
\hline URL & https://hdl. handle.net/11094/78509 \\
\hline rights & $\begin{array}{l}\text { Copyright (1993) by the American Physical } \\
\text { Society }\end{array}$ \\
\hline Note & \\
\hline
\end{tabular}

Osaka University Knowledge Archive : OUKA

https://ir. Library. osaka-u. ac. jp/

0saka University 


\title{
Thermal energy of the crystalline one-component plasma from dynamical simulations
}

\author{
R. T. Farouki and S. Hamaguchi \\ IBM Thomas J. Watson Research Center, P.O. Box 218, Yorktown Heights, New York 10598
}

(Received 5 February 1993)

\begin{abstract}
Molecular-dynamics simulations employing 1024 particles and a high-accuracy spline approximation of the Ewald potential have been used to measure the excess internal energy of the classical onecomponent plasma in both the fluid and solid (bcc) phases over a wide range $(1 \leq \Gamma \leq 2000)$ of the coupling parameter. The energy data for $\Gamma \leq 300$ are in excellent agreement with previous Monte Carlo calculations for this range, giving an independent corroboration of these calculations. Data in the crystalline regime $170 \leq \Gamma \leq 2000$ are used to estimate the coefficients of the anharmonic energy component by least-squares fits. The first coefficient is in reasonable agreement with peturbation-theory predictions, although it is argued that determination of the coefficients in this manner is a rather ill-conditioned problem. The free-energy curves of the fluid and solid phases are found to cross at $\Gamma_{m} \approx 173$, in agreement with recent estimates.
\end{abstract}

PACS number(s): 52.25.Kn, 52.65. $+\mathrm{z}, 64.30 .+\mathrm{t}$, 64.70.Dv

\section{INTRODUCTION}

The classical one-component plasma (OCP) is an idealized system of mobile ions of charge $Z e$, number density $n$, and temperature $T$, immersed in a neutralizing background of uniform charge density $\rho=-Z n e$. The OCP thermodynamics may be uniquely described in terms of the dimensionless coupling parameter

$$
\Gamma=\frac{(Z e)^{2}}{a k T},
$$

where $a=(3 / 4 \pi n)^{1 / 3}$ denotes the Wigner-Seitz radius; see [1-3] for reviews. The OCP "equation of state" has been the subject of extensive Monte Carlo (MC) calculations [4-13] employing the Ewald potential, which yields data pertinent to an infinite periodic system from simulations using only a finite number $N$ of particles confined to a cubical volume.

By fitting simple functional forms, guided by theory, to the measured excess (i.e., potential) energy per particle $U_{\text {ex }} / N k T$ for both fluid and solid phases of the OCP, it is possible to obtain the Helmholtz free energy as a function of $\Gamma$, and hence to deduce the pressure and entropy in terms of $\Gamma$. Furthermore, the intersection of the fluid and solid free-energy curves gives the value $\Gamma_{m}$ of the parameter (1) that identifies the phase transition.

The value of $\Gamma_{m}$ has an interesting history. In their pioneering study [4] Brush, Sahlin, and Teller observed phase changes of a 32-particle system at $\Gamma_{m} \approx 125$ using a rough approximation to the Ewald potential. Subsequently, Hansen [5] and Pollock and Hansen [6] deduced the value $\Gamma_{m}=155 \pm 10$ using a more sophisticated Ewald approximation and fitting of the excess energy to analytic expressions. However, DeWitt [7] doubted the accuracy of Hansen's data for large $\Gamma$, which motivated Slattery, Doolen, and DeWitt [8] to perform extensive new calculations with a very accurate potential.

Introducing a new functional form [14] for the fluid energy, they concluded that $\Gamma_{m}=168 \pm 4$ for $N=128$, add- ing in a note in proof that calculations with larger $N$ suggested the value $171 \pm 3$. Based on the same data, a later study [9] of the $N$ dependence gave $\Gamma_{m}=178 \pm 1$, but this prompted Ogata and Ichimaru [11] to question the "center-of-mass correction" for the fluid phase; with additional simulations they inferred that $\Gamma_{m}=180 \pm 1$.

Following [6], all these studies have assumed, in fitting the solid data, that the lowest-order anharmonic component of the lattice thermal energy is proportional to $T^{3}$. However, recent analytic work based on thermodynamic perturbation theory shows this assumption to be erroneous, and furnishes a numerical value for the coefficient of the anharmonic component proportional to $T^{2}$ [15-17]. Incorporating this lower-order term [17] reduces $\Gamma_{m}$ to $\sim 172$. In terms of empirical fits to the then-available MC data, which extended only to $\Gamma=300$, use of this term did not greatly improve the quality of fit or allow an independent verification of its coefficient.

However, DeWitt and co-workers had already independently observed $[12,13]$ that inclusion of such a term would give better agreement at $\Gamma=300$. More recently, DeWitt, Slattery, and Yang [18] have quoted MC results for the anharmonic energy at $\Gamma=400$ and 800 that appear to agree well with the predictions of [17]. In the present study, we attempt to fit anharmonic energy measurements over a wide range of $\Gamma$ values to two-, three-, and four-term expressions, and we assess the level of confidence in the computed coefficients in view of the fact that the anharmonic component is only a minuscule fraction of the total energy.

Our own interest in the OCP is as the formal limit of a model that we are developing to describe the thermodynamics of strongly coupled systems of charged particulates in plasmas $[19,20]$, based on dynamical simulations. We will report on this, incorporating the present OCP data, in due course.

\section{SIMULATION SCHEME}

We take for units of mass, length, and time, the ion mass $m$, the ion-sphere radius $a$, and $\sqrt{3} \omega^{-1}$, where 
$\omega=\left(4 \pi Z^{2} e^{2} n / m\right)^{1 / 2}$ is the ion plasma frequency. The equations of motion are then

$$
\frac{d^{2} \mathbf{r}_{k}}{d t^{2}}=-\sum_{j(\neq k)}^{N} \nabla \Phi\left(\mathbf{r}_{k}-\mathbf{r}_{j}\right)
$$

for $k=1, \ldots, N$, where $\Phi$ denotes the Ewald potential [4]

$$
\begin{aligned}
\Phi(\mathbf{r})= & \frac{\operatorname{erfc}(\sqrt{\pi} r / L)}{r}-\frac{1}{L} \\
& +\sum_{\mathbf{n}(\neq 0)}\left[\frac{\operatorname{erfc}(\sqrt{\pi}|\mathbf{r}+\mathbf{n} L| / L)}{|\mathbf{r}+\mathbf{n} L|}\right. \\
& \left.+\frac{\exp \left(-\pi|\mathbf{n}|^{2}\right) \cos (2 \pi \mathbf{n} \cdot \mathbf{r} / L)}{\pi|\mathbf{n}|^{2} L}\right]
\end{aligned}
$$

for the interaction of ions $k$ and $j$ at separation $\mathbf{r}=\mathbf{r}_{k}-\mathbf{r}_{j}$. Here erfc ( ) is the complementary error function, $L=(4 \pi N / 3)^{1 / 3}$ is the side length of the simulation volume in units of $a$, and $\mathbf{n}$ is a vector with integer components. The potential (3) is used in conjunction with the usual "minimum image" convention [21] for cubical periodic boundary conditions.

The infinite lattice sum in (3) was approximated in the simulations by evaluating it to a relative accuracy of $\sim 10^{-12}$ on a uniform grid of points and interpolating these values by a cubic $C^{2}$ tensor product spline function (using cubical symmetry to minimize computational and memory requirements). We used a true spline function, which has "smoothness" properties that are not matched by local Hermite interpolation schemes, such as that described in [10]. The approximation error diminishes in proportion to the fourth power of the sampling interval, so the accuracy is limited only by the available memory for storing the grid-point values.

However, tests showed that refining the grid beyond a certain point had no further discernible influence on thermodynamic variables. Consequently, we chose a grid density sufficient to guarantee a mean relative error $\sim 2 \times 10^{-7}$, and a maximum $<10^{-5}$, based on random sampling. Dynamical simulations require the gradient of the potential (3), and its derivatives, in computing forces between particles and in starting up the integration scheme; these are readily obtained from the spline representation. By careful optimization, it was possible to reduce the cost of computing the spline approximation for the anisotropic component of interparticle forces to a point where it was comparable to that of the isotropic term alone.

The equations of motion (2) were integrated using a predictor-corrector scheme in which the variation of the force on a particle over each time step is extrapolated from a quadratic fitting polynomial that interpolates its value at three preceding instances. Forces are obtained by pairwise summation over all particles, and the integration proceeds on an asynchronous time front with individually adjusted steps to ensure uniformly accurate trajectories under varying conditions. The integration commences using a high-order Taylor-series expansion for the accelerations $d^{2} \mathbf{r}_{k} / d t^{2}$, obtained by repeated symbolic differentiation of the right-hand side of Eq. (2).
To emulate averages over a canonical ensemble of fixed $\Gamma$ (temperature), we periodically synchronize all particles and renormalize their velocities [22] to bring the system kinetic energy into agreement with the target $\Gamma$ value. This also affords an opportunity to sample the system properties before restarting. Empirically, $\Delta t=1$ was found to be the optimum interval between successive temperature adjustments; mean thermodynamic properties were essentially unchanged upon adopting smaller intervals (which would degrade the program speed somewhat).

Fractional errors in the total system energy over each integration period were $\sim 10^{-4}$ at $\Gamma=1$, decreasing to $\sim 10^{-7}$ at $\Gamma=1000$. Thus, although at $\Gamma=1000$ the thermal motions represent only $\sim 0.1 \%$ of the total energy, this kinetic energy is nevertheless accurate to about four significant digits (the ratio of the actual system potential and kinetic energies differs from $\Gamma$ by a numerical factor that is $\sim 2.5$ at $\Gamma=1$ and $\sim 1.7$ at $\Gamma=1000$ ).

$N=1024$ in all simulations reported here; residual small-number effects are expected to be negligible. Most runs began with the particles on a bcc lattice and a Maxwellian velocity distribution corresponding to the target $\Gamma$. All cases with $\Gamma<160$ melted, in agreement with the observations of [8]. For $\Gamma<100$ the melting was very sudden, and the system rapidly lost "memory" of its initial crystalline configuration. For $\Gamma \geq 100$, however, melting was slower (but still within the first 100 time units) and the final excess energy seemed to be slightly influenced by the initial configuration. Therefore cases with $100 \leq \Gamma \leq 180$ were repeated with random initial configurations. All runs were evolved for at least 300 time units, allowing the system to equilibrate to the desired $\Gamma$ value for the first 100 or 200 units before averaging its properties over an additional 100 or 200 units.

We do not report here on simulations of "freezing" of the OCP at $\Gamma>\Gamma_{m}$ from random initial configurations. The time scale for this is typically much larger than that for melting and the resulting crystalline form is often quite defective, even if $N$ is exactly compatible with a bcc or fcc lattice aligned with the simulation volume [18,23]. Correspondingly, the measured energy is not representative of the perfect crystalline phase.

\section{THERMODYNAMIC MEASUREMENTS}

Each time the OCP simulation is synchronized for temperature adjustment, the excess energy per ion in units of $k T$ is computed using the expression [4]

$$
\frac{U_{\mathrm{ex}}}{N k T}=\Gamma\left[\frac{E_{m}}{2}+\frac{1}{N} \sum_{j=1}^{N-1} \sum_{k=j+1}^{N} \Phi\left(\mathbf{r}_{j}-\mathbf{r}_{k}\right)\right],
$$

where

$$
E_{m}=\lim _{\mathbf{r} \rightarrow 0}\left(\Phi(\mathbf{r})-\frac{1}{r}\right) \approx \frac{-2.837297479}{L} .
$$

After settling, averages of 100 or 200 consecutive measurements of (4) are obtained, together with rms dispersions. 
In Table I we compare data for the range $1 \leq T \leq 300$, averaged over $200<t \leq 300$, with the MC data of [8]. Here "type" indicates the number of particles, and either a random $(L)$ or bcc lattice $(S)$ initial configuration. A1though the quoted uncertainties for our data exceed those of [8] by more than an order of magnitude, the agreement between the data sets is evidently much better than would be expected on the basis of these "errors." In our dynamical simulations, the measured rms energy deviations largely represent true thermodynamic fluctuations for an $N=1024$ system, and cannot be made arbitrarily small (as with the MC method) by sufficient averaging.

Thus it would be overly pessimistic to interpret the rms fluctuations given in Table $I$ as estimates of the actual uncertainty in our nominal energy values. Since we have no means of deducing more realistic error bounds on our energy data, we shall use only unweighted least-squares fits to this data. The most significant discrepancies in Table I occur at $\Gamma=160$, near the phase transition, where largescale fluctuations become important.

The thermal part of the excess energy is defined by

$$
\frac{U_{\mathrm{th}}}{N k T}=\frac{U_{\mathrm{ex}}-U_{0}}{N k T},
$$

where

$$
\frac{U_{0}}{N k T} \approx-0.895929256 \Gamma
$$

represents the Madelung energy of a zero-temperature bcc lattice. With the MC method, it is customary-for the solid phase, at least [11] - to multiply (6) by the factor $N /(N-1)$ to correct for motion of the system center of mass. This correction is superfluous in dynamical simulations, where (apart from minuscule integration errors) the center of mass is immobile.

Following [12], we have computed (unweighted) leastsquares fits of our fluid data for $1 \leq \Gamma \leq 150$ to the form

$$
\frac{U_{\mathrm{ex}}}{N k T}=a \Gamma+b \Gamma^{s}+c+d \Gamma^{-s}
$$

for various exponents $s$. As a measure for the quality of the fit we use $\sigma$, the square root of the sample variance [24]. Data for $160 \leq \Gamma \leq 180$ were excluded from these fits, as they are conspicuously more noisy than those for $\Gamma<160$.

Table II shows the computed coefficients of (8) for various exponents $s$; compare with Table II of [12]. We find

\begin{tabular}{|c|c|c|c|c|}
\hline \multirow[b]{2}{*}{$\Gamma$} & \multicolumn{2}{|c|}{ SDD } & \multicolumn{2}{|c|}{ Present results } \\
\hline & $U_{\mathrm{ex}} / N k T$ & Type & $U_{\mathrm{ex}} / N k T$ & Type \\
\hline 1 & $-0.572 \pm 0.000$ & $686 L$ & $-0.572 \pm 0.012$ & $1024 S$ \\
\hline 2 & $-1.320 \pm 0.000$ & $686 L$ & $-1.322 \pm 0.012$ & $1024 S$ \\
\hline 5 & $-3.757 \pm 0.000$ & $686 L$ & $-3.755 \pm 0.013$ & $1024 S$ \\
\hline 10 & $-7.998 \pm 0.001$ & $686 L$ & $-8.002 \pm 0.020$ & $1024 S$ \\
\hline 15 & $-12.318 \pm 0.001$ & $686 L$ & $-12.311 \pm 0.021$ & $1024 S$ \\
\hline 20 & $-16.673 \pm 0.001$ & $686 L$ & $-16.668 \pm 0.023$ & $1024 S$ \\
\hline 30 & $-25.441 \pm 0.001$ & $686 \bar{L}$ & $-25.438 \pm 0.022$ & $1024 S$ \\
\hline 40 & $-34.255 \pm 0.001$ & $686 L$ & $-34.258 \pm 0.027$ & $1024 S$ \\
\hline 50 & $-43.102 \pm 0.001$ & $686 L$ & $-43.093 \pm 0.027$ & $1024 S$ \\
\hline 60 & $-51.961 \pm 0.001$ & $686 \bar{L}$ & $-51.963 \pm 0.021$ & $1024 S$ \\
\hline 80 & $-69.725 \pm 0.001$ & $686 L$ & $-69.727 \pm 0.031$ & $1024 S$ \\
\hline 100 & $-87.522 \pm 0.001$ & $1024 L$ & $-87.524 \pm 0.026$ & $1024 L$ \\
\hline 100 & & & $-87.532 \pm 0.029$ & $1024 S$ \\
\hline 120 & $-105.345 \pm 0.002$ & $1024 L$ & $-105.342 \pm 0.030$ & $1024 L$ \\
\hline 120 & & & $-105.335 \pm 0.031$ & $1024 S$ \\
\hline 140 & $-123.188 \pm 0.002$ & $1024 L$ & $-123.188 \pm 0.034$ & $1024 L$ \\
\hline 140 & & & $-123.194 \pm 0.030$ & $1024 S$ \\
\hline 150 & $-132.110 \pm 0.002$ & $1024 L$ & $-132.102 \pm 0.031$ & $1024 L$ \\
\hline 150 & & & $-132.104 \pm 0.033$ & $1024 S$ \\
\hline 160 & $-141.036 \pm 0.002$ & $1024 L$ & $-141.055 \pm 0.030$ & $1024 L$ \\
\hline 160 & $-141.716 \pm 0.009$ & $250 S$ & $-141.666 \pm 0.049$ & $1024 S$ \\
\hline 170 & $-149.970 \pm 0.001$ & $1024 L$ & $-149.972 \pm 0.034$ & $1024 L$ \\
\hline 170 & $-150.697 \pm 0.002$ & $686 S$ & $-150.698 \pm 0.031$ & $1024 S$ \\
\hline 180 & & & $-158.894 \pm 0.037$ & $1024 L$ \\
\hline 180 & $-159.667 \pm 0.002$ & $432 S$ & $-159.670 \pm 0.033$ & $1024 S$ \\
\hline 190 & & & $-168.638 \pm 0.030$ & $1024 S$ \\
\hline 200 & $-177.603 \pm 0.001$ & $686 S$ & $-177.606 \pm 0.037$ & $1024 S$ \\
\hline 220 & $-195.536 \pm 0.002$ & $432 S$ & $-195.534 \pm 0.032$ & $1024 S$ \\
\hline 240 & $-213.463 \pm 0.001$ & $686 S$ & $-213.461 \pm 0.027$ & $1024 S$ \\
\hline 260 & & & $-231.385 \pm 0.028$ & $1024 S$ \\
\hline 280 & & & $-249.309 \pm 0.029$ & $1024 S$ \\
\hline 300 & $-267.233 \pm 0.001$ & $686 S$ & $-267.233 \pm 0.032$ & $1024 S$ \\
\hline
\end{tabular}

TABLE I. Comparison of the present results for the OCP excess energy for $1 \leq \Gamma \leq 300$ with those of Ref. [8]. 
TABLE II. Coefficients of least-squares fits to fluid energy data. The column headings $a, b, c$, and $d$ corrrespond to Table II of Ref. [12].

\begin{tabular}{ccccrc}
\hline \hline$s$ & $a$ & $b$ & $c$ & \multicolumn{1}{c}{$d$} & $\sigma$ \\
\hline 0.25 & -0.89813 & 0.98686 & -0.91095 & 0.25098 & 0.0036 \\
0.30 & -0.89879 & 0.69350 & -0.41725 & 0.04977 & 0.0036 \\
$\frac{1}{3}$ & -0.89932 & 0.56484 & -0.20388 & -0.03555 & 0.0039 \\
0.35 & -0.89962 & 0.51347 & -0.11926 & -0.06912 & 0.0041 \\
0.40 & -0.90065 & 0.39525 & 0.07447 & -0.14555 & 0.0052 \\
0.50 & -0.90353 & 0.25581 & 0.30315 & -0.23631 & 0.0086 \\
\hline
\end{tabular}

that values $s$ between 0.25 and 0.35 give fits of essentially equal quality: our data to not particularly favor $s=\frac{1}{3}$ over $s=\frac{1}{4}$, as suggested by Stringfellow, DeWitt, and Slattery [12], and our $\sigma$ values are only slightly larger than theirs (including the data for $160 \leq \Gamma \leq 180$ does not influence the relative quality of the fits, although all are somewhat degraded). Since it has a theoretical basis [14] and performs as well as any other, we prefer the $s=\frac{1}{4}$ fit.

As seen in Table I, our solid energy data for $170 \leq \Gamma \leq 300$ are also in excellent agreement with that of [8]. Further simulations with $\Gamma$ up to 2000 have been performed: we present data for the excess thermal energy (6) of the solid phase for the entire range $170 \leq \Gamma \leq 2000$ in Table III. Since this thermal energy is just a small fraction of the static energy (7), we have used averaging over $100<t \leq 300$ to reduce noise. For the cases $\Gamma=170$, 180 , and 190 , the system had not fully settled by $t=100$, so it was evolved for an additional 100 units of time and averaged over $200<t \leq 400$.

We exclude data at $\Gamma \leq 160$ in considering the solid energy because of large fluctuations in this regime, and fit to

TABLE III. Thermal part of crystalline OCP excess energy for $170 \leq \Gamma \leq 2000$.

\begin{tabular}{ccc}
\hline \hline$\Gamma$ & $U_{\text {th }} / N k T$ & Type \\
\hline 170 & 1.6089 & $1024 S$ \\
180 & 1.5987 & $1024 S$ \\
190 & 1.5894 & $1024 S$ \\
200 & 1.5823 & $1024 S$ \\
220 & 1.5715 & $1024 S$ \\
240 & 1.5620 & $1024 S$ \\
260 & 1.5564 & $1024 S$ \\
280 & 1.5508 & $1024 S$ \\
300 & 1.5453 & $1024 S$ \\
320 & 1.5417 & $1024 S$ \\
350 & 1.5375 & $1024 S$ \\
400 & 1.5311 & $1024 S$ \\
450 & 1.5265 & $1024 S$ \\
500 & 1.5249 & $1024 S$ \\
550 & 1.5211 & $1024 S$ \\
600 & 1.5194 & $1024 S$ \\
700 & 1.5154 & $1024 S$ \\
800 & 1.5129 & $1024 S$ \\
900 & 1.5114 & $1024 S$ \\
1000 & 1.5103 & $1024 S$ \\
1200 & 1.5087 & $1024 S$ \\
1500 & 1.5066 & $1024 S$ \\
2000 & 1.5044 & $1024 S$ \\
\hline \hline
\end{tabular}

the form [17]

$$
\frac{U_{\mathrm{th}}}{N k T}=\frac{3}{2}+\frac{A_{1}}{\Gamma}+\frac{A_{2}}{\Gamma^{2}}+\frac{A_{3}}{\Gamma^{3}}+\cdots,
$$

where $\frac{3}{2}$ is the harmonic component of the thermal excess energy and the terms with coefficients $A_{1}, A_{2}, A_{3}, \ldots$ represent anharmonic contributions.

In their early study [6], Pollock and Hansen argued that $A_{1}$ is identically zero and they neglected the $A_{3}$ and higher terms in (9). This assumption was adopted by all MC studies [6-11] up to [12], and for $\Gamma \lesssim 300$ it can give reasonable empirical fits to the energy data. However, it is completely inconsistent with our extended data for $\Gamma \gtrsim 300$ in Table III. To illustrate this, Fig. 1 compares a three-term least-squares fit of the form [9] to our solid data (solid curve) with one assuming that only $A_{2} \neq 0$ (dashed curve).

The numerical coefficients of the three-term fit are found to be

$$
\begin{aligned}
& A_{1}=9.65 \pm 0.32, \quad A_{2}=840 \pm 171, \\
& A_{3}=(1.101 \pm 0.216) \times 10^{5}
\end{aligned}
$$

and the square root of the corresponding sample variance

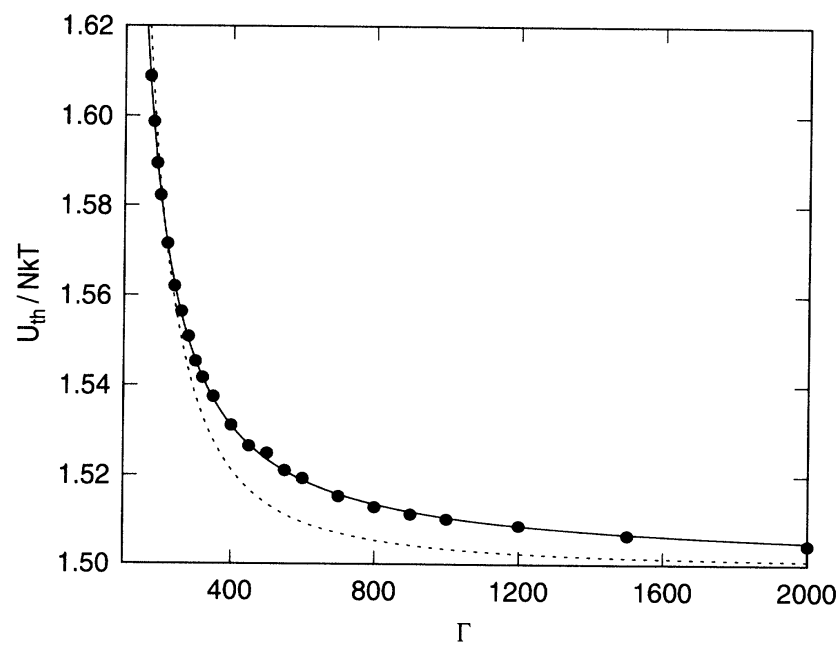

FIG. 1. Thermal energy of crystalline OCP for $170 \leq \Gamma$ $\leq 2000$ from the simulations (circles) and a three-term fit (solid curve) of the form (9). The dashed curve shows how poorly a one-term fit with only $A_{2} \neq 0$ performs. 
TABLE IV. Coefficients of two-term fits for various ranges of $\Gamma$.

\begin{tabular}{ccccc}
\hline \hline Range & No. & $A_{1}$ & $A_{2}$ & $\sigma$ \\
\hline $170 \leq \Gamma \leq 400$ & 12 & 7.65 & 1806 & 0.0009 \\
$170 \leq \Gamma \leq 800$ & 18 & 8.12 & 1711 & 0.0010 \\
$170 \leq \Gamma \leq 2000$ & 23 & 8.19 & 1697 & 0.0009 \\
$400 \leq \Gamma \leq 2000$ & 12 & 8.82 & 1511 & 0.0005 \\
$800 \leq \Gamma \leq 2000$ & 6 & 9.09 & 1088 & 0.0003 \\
\hline \hline
\end{tabular}

is $\sigma \approx 0.0006$. The uncertainties are based on standard estimates [24] - see also the Appendix - assuming that the measured energy values have uniform errors of order $\sigma$.

The above $A_{1}$ values agrees reasonably well with the perturbation-theory predictions: 10.9 [16] and 10.84 (for $N \rightarrow \infty$ ) [17], though not with the value 3.65 of [15]. However, it should be noted that these coefficients are quite sensitive to small changes in the data of Table III, and we believe the quoted errors are probably too optimistic; see the Appendix.

Furthermore, two- and four-term expressions of the form (9) give fits of comparable quality with rather different values. For the former, we obtain

$$
A_{1}=8.19 \pm 0.20, \quad A_{2}=1697 \pm 44
$$

with $\sigma=0.0009$, and for the latter

$$
\begin{aligned}
& A_{1}=8.18 \pm 0.52, \quad A_{2}=2396 \pm 493, \\
& A_{3}=(-3.614 \pm 1.444) \times 10^{5}, \\
& A_{4}=(4.296 \pm 1.306) \times 10^{7},
\end{aligned}
$$

giving a slightly smaller dispersion, $\sigma=0.0005$, than the three-term fit.

Overall, we favor the three-term fit. It gives an appreciable reduction in $\sigma$ over just two terms, while inclusion of a fourth term yields marginal further improvement, and its coefficients appear to have larger uncertainties (see also the Appendix). Nevertheless, it should be borne in mind that the computed values (10) may differ somewhat from theoretical predictions on account of their trying to "compensate" for the omission of higher-order terms. Fixing $A_{1}=10.84$ [17] in the three-term fit gives $A_{2}=221$ and $A_{3}=1.837 \times 10^{5}$ with $\sigma=0.0008$, somewhat higher than the unconstrained fit (10).

We have also tried fitting to subsets of the data in Table III for various ranges in $\Gamma$. If the series (9) is convergent, the first term will dominate at sufficiently large $\Gamma$, and one expects that the most accurate determination of $A_{1}$ could be obtained by fitting to data at large $\Gamma$
TABLE V. Coefficients of three-term fits for various ranges of $\Gamma$.

\begin{tabular}{crrrcc}
\hline \hline Range & No. & \multicolumn{1}{c}{$A_{1}$} & $A_{2}$ & \multicolumn{1}{c}{$A_{3}$} & $\sigma$ \\
\hline $170 \leq \Gamma \leq 400$ & 12 & 10.90 & 247 & $1.765 \times 10^{5}$ & 0.0005 \\
$170 \leq \Gamma \leq 800$ & 18 & 10.02 & 654 & $1.314 \times 10^{5}$ & 0.0006 \\
$170 \leq \Gamma \leq 2000$ & 23 & 9.65 & 840 & $1.101 \times 10^{5}$ & 0.0006 \\
\hline \hline
\end{tabular}

values only, provided they are adequate in quantity and quality. [If one accepts the values (10), the $A_{2}$ and $A_{3}$ terms contribute nearly $50 \%$ of the anharmonic energy at $\Gamma=170$, but less than $5 \%$ at $\Gamma=2000$.]

Results for two-, three-, and four-term fits are shown in Tables IV-VI. In the three-term fits, a tendency for $A_{1}$ to decrease away from the perturbation-theory prediction is evident as one includes data at larger $\Gamma$, while the opposite seems to be true for the two- and four-term fits. However, as we shall argue in the Appendix, it is not clear whether these trends can be regarded as significant. Three- and four-term fits to the $400 \leq \Gamma \leq 2000$ and $800 \leq \Gamma \leq 2000$ data resulted in an erratic behavior of the coefficients (although the $\sigma$ values were quite small), most likely because the $A_{3}$ and $A_{4}$ terms cannot be reliably determined in this range.

\section{DISCUSSION}

Figure 2 shows the fitted thermal energies for the fluid and solid phases for the range $1 \leq \Gamma \leq 350$. The corresponding excess Helmholtz free energies are defined by the integral [5]

$$
\frac{F_{\mathrm{ex}}}{N k T}=\int_{0}^{\Gamma} \frac{U_{\mathrm{ex}}}{N k T} \frac{d \Gamma^{\prime}}{\Gamma^{\prime}} .
$$

Thus, for the forms (8) and (9), adding the ideal-gas contribution gives [12] the total free energy

$$
\begin{aligned}
\frac{F}{N k T}= & a \Gamma+\frac{b \Gamma^{s}-d \Gamma^{-s}}{s}+(3+c) \ln \Gamma \\
& -\left(a+\frac{b-d}{s}+1.1516\right)
\end{aligned}
$$

for the fluid phase, and

$$
\begin{aligned}
\frac{F}{N k T}= & -0.895929256 \Gamma+4.5 \ln \Gamma \\
& -\frac{A_{1}}{\Gamma}-\frac{A_{2}}{2 \Gamma^{2}}-\frac{A_{3}}{3 \Gamma^{3}}-1.8856
\end{aligned}
$$

for the solid phase (the integration constants are from [12]).

TABLE VI. Coefficients of four-term fits for various ranges of $\Gamma$.

\begin{tabular}{ccccccc}
\hline \hline Range & No & $A_{1}$ & $A_{2}$ & $A_{3}$ & $A_{4}$ & $\sigma$ \\
\hline $170 \leq \Gamma \leq 400$ & 12 & 5.98 & 3911 & $-6.987 \times 10^{5}$ & $6.740 \times 10^{7}$ & 0.0004 \\
$170 \leq \Gamma \leq 800$ & 18 & 8.06 & 2502 & $-3.892 \times 10^{5}$ & $4.525 \times 10^{7}$ & 0.0005 \\
$170 \leq \Gamma \leq 2000$ & 23 & 8.18 & 2396 & $-3.614 \times 10^{5}$ & $4.296 \times 10^{7}$ & 0.0005 \\
\hline \hline
\end{tabular}




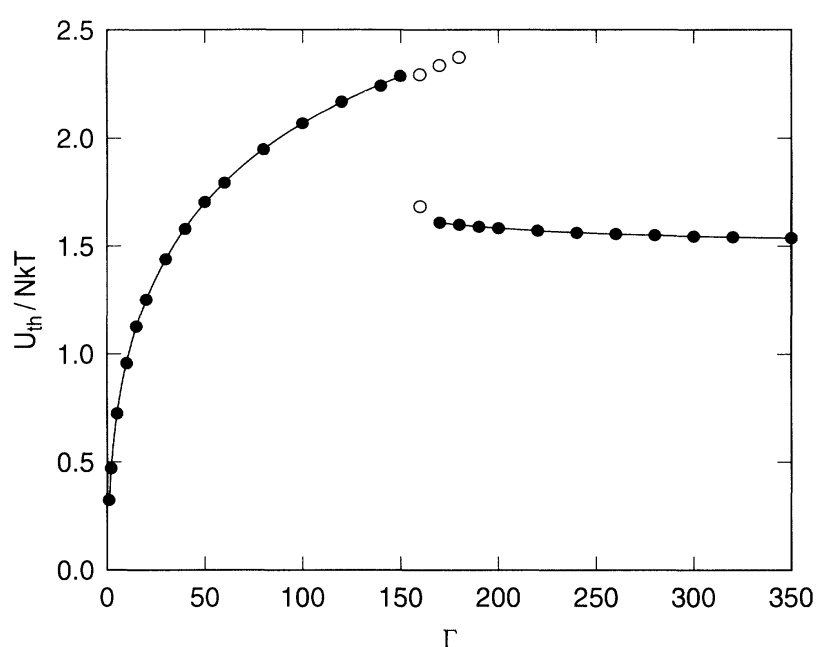

FIG. 2. Fitted thermal excess energies for the fluid and solid OCP phases. Open circles represent values that are omitted from the fits.

Using the $s=\frac{1}{4}$ fit to the fluid data and the three-term fit to the entire range of solid data, we find that the freeenergy curves cross at $\Gamma_{m} \approx 173$, in reasonable agreement with the value determined in [17] using the theoretical coefficient $A_{1}=10.84$ together with MC data taken from [9].

In conclusion, we have shown that molecular dynamics can be used to measure the OCP internal energy over a wide range of $\Gamma$ to an accuracy approaching that of the MC data, although the averaging is over far fewer configurations. A large range of energy measurements for the solid phase was made in order to estimate the anharmonic coefficients by least-squares fits. A threeterm fit gives better results for $170 \leq \Gamma \leq 2000$ than a two-term fit, but with an $A_{1}$ value some $\sim 10 \%$ lower than perturbation-theory predictions $[16,17]$. In view of the sensitive nature of the fitting process (see the Appendix), however, this discrepancy may not be significant. Fits with four or more terms are not warranted by the quantity and quality of the data.

Note added in proof. Further simulations have been performed at large $\Gamma$ using an Ewald potential approximation an order of magnitude more accurate than that used initially. Energy values obtained from these runs were found to be systematically slightly higher than listed in Table III (the anharmonic component was a few percent higher at $\Gamma=300$ and $\gtrsim 10 \%$ higher for $\Gamma \geq 1000$ ). A two-term fit to 12 new anharmonic energy values for $300 \leq \Gamma \leq 3200$ gives $A_{1}=11.1 \pm 0.2$ and $A_{2}=810 \pm 68$.

\section{ACKNOWLEDGMENTS}

We wish to thank H. E. DeWitt and E. E. Salpeter for encouragement and helpful discussions.

\section{APPENDIX: LEAST-SQUARES SENSITIVITY ANALYSIS}

The anharmonic component diminishes from $\sim 0.1 \%$ of the total energy at $\Gamma=170$ to $\sim 0.001 \%$ at $\Gamma=1000$, so if an accuracy of $1 \%$ in its value is desired, the total energy must be known to a relative accuracy of between $10^{-5}$ and $10^{-7}$ over this range. Although our data are nominally of such an accuracy, we argue here that the least-squares procedure for determining the anharmonic coefficients $A_{1}, A_{2}, \ldots$ is intrinsically rather ill conditioned (i.e., the values of these coefficients can be sensitive to small perturbations in the energy values), and thus a measurement accuracy of $1 \%$ for the anharmonic energy may be inadequate for a reliable estimation of its coefficients.

We begin by rewriting (9), for an $m$-term fit, in the form

$$
u(\gamma)=\sum_{k=1}^{m} a_{k} \gamma^{-k}
$$

where $u=U_{\text {th }} / N k T-\frac{3}{2}, \gamma=\Gamma / \Gamma_{c}$, and the "characteristic" value $\Gamma_{c}$ will be chosen so as to give commensurate reduced coefficients $a_{k}=A_{k} / \Gamma_{c}^{k}$ [with $\Gamma_{c}=100$ the values (10) yield $a_{1} \approx 0.097, a_{2} \approx 0.084$, and $a_{3} \approx 0.110$ ].

If $u_{1}, \ldots, u_{n}$ are the anharmonic energies measured at $n$ values $\gamma_{1}, \ldots, \gamma_{n}$, we wish to minimize the sum

$$
\chi^{2}=\sum_{i=1}^{n}\left[u_{i}-\sum_{k=1}^{m} a_{k} \gamma_{i}^{-k}\right]^{2} \text {. }
$$

Setting the derivatives of $\chi^{2}$ with respect to $a_{1}, \ldots, a_{m}$ equal to zero gives the normal equations

$$
\sum_{k=1}^{m}\left[\sum_{i=1}^{n} \gamma_{i}^{-(j+k)}\right] a_{k}=\sum_{i=1}^{n} \gamma_{i}^{-j} u_{i}
$$

for $j=1, \ldots, m$. In terms of the $n \times m$ Vandermonde matrix $\mathbf{S}$ with elements

$$
S_{\alpha \beta}=\gamma_{\alpha}^{-\beta}
$$

and the vectors $\mathbf{a}=\left(a_{1}, \ldots, a_{m}\right)^{T}$ and $\mathbf{u}=\left(u_{1}, \ldots, u_{n}\right)^{T}$, Eq. (A3) can be written in matrix form as

$$
\left(\mathbf{S}^{T} \mathbf{S}\right) \mathbf{a}=\mathbf{S}^{T} \mathbf{u} \text {. }
$$

The formal solution for the coefficients is then simply

$$
\mathbf{a}=\left(\mathbf{S}^{T} \mathbf{S}\right)^{-1} \mathbf{S}^{T} \mathbf{u} \text {. }
$$

Once $a_{1}, \ldots, a_{m}$ are determined, a numerical value can be computed for (A2) and the sample variance for the fit is given in terms of this value [24] by $\sigma^{2}=\chi^{2} /(n-m)$. Further, if the measurement errors for $u$ are uncorrelated, the standard estimates for the probable uncertainties $\delta a_{1}, \ldots, \delta a_{m}$ in the computed coefficients are [24]

$$
\left(\delta a_{j}\right)^{2}=\sum_{i=1}^{n}\left(\frac{\partial a_{j}}{\partial u_{i}}\right)^{2} \sigma^{2}=\mathscr{E}_{j} \sigma^{2}
$$

for $j=1, \ldots, m$, where $\mathscr{E}_{j}$ proves to be the $j$ th diagonal element of $\left(\mathbf{S}^{T} \mathbf{S}\right)^{-1}$ (the error bounds quoted in Sec. III above are based on this formula).

As is well known, the normal equations (A3) can be quite ill conditioned, and unsophisticated solution procedures may induce large arithmetical errors. However, we have verified the accuracy of our solutions by representing the $\gamma_{1}, \ldots, \gamma_{n}$ and $u_{1}, \ldots, u_{n}$ values to high accuracy as rational fractions and solving for $a_{1}, \ldots, a_{m}$ in exact rational arithmetic. What concerns 
TABLE VII. Norms of least-squares matrices $(170 \leq \Gamma$ $\leq 2000$ ).

\begin{tabular}{lrrr}
\hline & $p$ & 2 & \multicolumn{1}{c}{$\infty$} \\
\hline 2 & 1 & & \\
3 & 2.93 & 28.2 & 268.9 \\
4 & 5.48 & 46.9 & 415.4 \\
\hline \hline
\end{tabular}

us here is the potential inflation of the $\sim 1 \%$ measurement errors in $u_{1}, \ldots, u_{n}$ that this ill condition may incur in determining the coefficients $a_{1}, \ldots, a_{m}$.

Since the matrix $\left(\mathbf{S}^{T} \mathbf{S}\right)^{-1} \mathbf{S}^{T}$ that defines the leastsquares relationship (A5) between the measured energies $\mathbf{u}$ and the coefficients $\mathbf{a}$ is not square, the usual characterization of the fractional sensitivity of the latter to the former in terms of a matrix condition number is not possible. However, the norm of this matrix gives a bound on the magnitude of the absolute errors $\delta \mathbf{a}$ $=\left(\delta a_{1}, \ldots, \delta a_{m}\right)^{T}$ in the coefficients due to measurement errors $\delta \mathbf{u}=\left(\delta u_{1}, \ldots, \delta u_{n}\right)^{T}$ in the energy. From (A5) we have

$$
\delta \mathbf{a}=\left(\mathbf{S}^{T} \mathbf{S}\right)^{-1} \mathbf{S}^{T} \delta \mathbf{u},
$$

and by the definitions

$$
\|\mathbf{x}\|_{p}=\left[\sum_{i=1}^{n}\left|x_{i}\right|^{p}\right]^{1 / p}
$$

and

$$
\|\mathbf{M}\|_{p}=\sup _{\mathbf{x} \neq 0} \frac{\|\mathbf{M} \mathbf{x}\|_{p}}{\|\mathbf{x}\|_{p}}
$$

of the $n$-element vector norm $\|\mathbf{x}\|_{p}$ and the "subordinate" $m \times n$ matrix norm $\|\mathbf{M}\|_{p}$, we have

$$
\|\delta \mathbf{a}\|_{p} \leq\left\|\left(\mathbf{S}^{T} \mathbf{S}\right)^{-1} \mathbf{S}^{T}\right\|_{p}\|\delta \mathbf{u}\|_{p}
$$

Note that the above bound on $\|\delta \mathbf{a}\|_{p}$ is sharp, i.e., there exists a $\delta \mathbf{u}$ for which it will be attained.

Only the $p=1,2$, and $\infty$ matrix norms (where $\left.\|\mathbf{x}\|_{\infty}=\max _{i}\left|x_{i}\right|\right)$ admit simple evaluation: $\|\mathbf{M}\|_{1}$ and $\|\mathbf{M}\|_{\infty}$ are the greatest of the sums of absolute values of the matrix elements across columns and rows, respectively, while $\|\mathbf{M}\|_{2}^{2}$ is the largest eigenvalue of the symmetric matrix $\mathbf{M}^{T} \mathbf{M}$ [25]. Table VII lists computed norms for the least-squares matrix $\left(\mathbf{S}^{T} \mathbf{S}\right)^{-1} \mathbf{S}^{T}$ for cases where all 23 $\gamma$ values in Table III are used for fits with $m=2,3$, or 4 terms.

Thus estimates of the worst-case mean, rms, and maximum errors in the coefficients $a_{1}, \ldots, a_{m}$ may be obtained from these norms by setting

$$
\begin{aligned}
& \|\delta \mathbf{a}\|_{1} \approx m|\delta a|_{\text {mean }}, \quad\|\delta \mathbf{a}\|_{2} \approx \sqrt{m}(\delta a)_{\mathrm{rms}}, \\
& \|\delta \mathbf{a}\|_{\infty} \approx|\delta a|_{\max }
\end{aligned}
$$

and

$$
\|\delta \mathbf{u}\|_{1} \approx n \sigma, \quad\|\delta \mathbf{u}\|_{2} \approx \sqrt{n} \sigma, \quad\|\delta \mathbf{u}\|_{\infty} \approx \sigma
$$

in (A9). For the $p=2$ norm, for example, we have $\sigma=0.0006$ for a fit with $m=3$ terms to all the values in Table III, and hence $(\delta a)_{\text {rms }} \lesssim 0.078$-in this case, the error bound is comparable to the nominal values $\left(a_{1}, a_{2}, a_{3}\right) \approx(0.097,0.084,0.110)$ of the coefficients (for the error bounds on $A_{1}, A_{2}, A_{3}$, multiply 0.078 by $\left.\Gamma_{c}, \Gamma_{c}^{2}, \Gamma_{c}^{3}\right)$. The situation is somewhat better with twoterm fits, but worse with four-term fits. Thus the uncertainties quoted in Eqs. (10) -(12) should be regarded with a degree of skepticism.
[1] M. Baus and J.-P. Hansen, Phys. Rep. 59, 1 (1980).

[2] S. Ichimaru, Rev. Mod. Phys. 54, 1017 (1982).

[3] S. Ichimaru, H. Iyetomi, and S. Tanaka, Phys. Rep. 149, 91 (1987).

[4] S. G. Brush, H. L. Sahlin, and E. Teller, J. Chem. Phys. 45, 2102 (1966).

[5] J.-P. Hansen, Phys. Rev. A 8, 3096 (1973).

[6] E. L. Pollock and J.-P. Hansen, Phys. Rev. A 8, 3110 (1973).

[7] H. E. DeWitt, Phys. Rev. A 14, 1290 (1976).

[8] W. L. Slattery, G. D. Doolen, and H. E. DeWitt, Phys. Rev. A 21, 2087 (1980).

[9] W. L. Slattery, G. D. Doolen, and H. E. DeWitt, Phys. Rev. A 26, 2255 (1982).

[10] H. L. Helfer, R. L. McCrory, and H. M. van Horn, J. Stat. Phys. 37, 577 (1984).

[11] S. Ogata and S. Ichimaru, Phys. Rev. A 36, 5451 (1987).

[12] G. C. Stringfellow, H. E. DeWitt, and W. L. Slattery, Phys. Rev. A 41, 1105 (1990).

[13] H. E. DeWitt, W. L. Slattery, and G. C. Stringfellow, in Strongly Coupled Plasma Physics, edited by S. Ichimaru (Elsevier, Amsterdam, 1990).

[14] H. E. DeWitt and Y. Rosenfeld, Phys. Lett. 75A, 79
(1979).

[15] R. C. Albers and J. E. Gubernatis, Phys. Rev. B 33, 5180 (1986).

[16] H. Nagara, Y. Nagata, and T. Nakamura, Phys. Rev. A 36, 1859 (1987).

[17] D. H. E. Dubin, Phys. Rev. A 42, 4972 (1990).

[18] H. E. DeWitt, W. L. Slattery, and J. Yang, in Proceedings of the International Conference on the Physics of Strongly Coupled Plasmas, edited by H. M. Van Horn and S. Ichimaru (University of Rochester Press, Rochester, NY, 1993).

[19] H. Ikezi, Phys. Fluids 29, 1764 (1986).

[20] R. T. Farouki and S. Hamaguchi, Appl. Phys. Lett. 61, 2973 (1992).

[21] M. P. Allen and D. J. Tildesley, Computer Simulation of Liquids (Oxford University Press, Oxford, 1987).

[22] F. F. Abraham, Adv. Phys. 35, 1 (1986).

[23] S. Ogata, Phys. Rev. A 45, 1122 (1992).

[24] P. R. Bevington, Data Reduction and Error Analysis for the Physical Sciences (McGraw-Hill, New York, 1969).

[25] G. W. Stewart, Introduction to Matrix Computations (Academic, New York, 1973). 